\title{
A minimalist model of characteristic earthquakes
}

\author{
M. Vázquez-Prada, Á. González, J. B. Gómez, and A. F. Pacheco \\ Faculty of Sciences, University of Zaragoza, Pedro Cerbuna, 12, 50009 Zaragoza, Spain
}

Received: 3 May 2002 - Revised: 30 July 2002 - Accepted: 31 July 2002

\begin{abstract}
In a spirit akin to the sandpile model of selforganized criticality, we present a simple statistical model of the cellular-automaton type which simulates the role of an asperity in the dynamics of a one-dimensional fault. This model produces an earthquake spectrum similar to the characteristic-earthquake behaviour of some seismic faults. This model, that has no parameter, is amenable to an algebraic description as a Markov Chain. This possibility illuminates some important results, obtained by Monte Carlo simulations, such as the earthquake size-frequency relation and the recurrence time of the characteristic earthquake.
\end{abstract}

\section{Introduction}

If there is a well-established fact about regional seismicity it is the relationship between the magnitude of an earthquake and its frequency, known as the Gutenberg-Richter law (Gutenberg and Richter, 1956). This law is of the power-law type when magnitudes are expressed in terms of rupture area

$N \propto S^{-b}$,

where $N$ is the number of observed earthquakes with rupture area greater than $S$, and $b$ is the so-called $b$-value, which is a "universal constant" in the range 0.5-1.5 (Kanamori and Anderson, 1975; Kagan, 1999; Turcotte, 1999). The GutenbergRichter law implies that earthquakes are, on a regional or world-wide scale, a self-similar phenomenon lacking a characteristic scale (but see Knopoff, 2000).

It is important to notice, however, that the GutenbergRichter law is a property of regional seismicity, appearing when we average seismicity over big enough areas and long enough time intervals. In the last ten years, a wealth of data has been collected to extract statistics on individual systems of earthquake faults (Wesnousky, 1994; Sieh, 1996; Petersen et al., 1996). Interestingly, it has been found that the distribution of earthquake magnitudes may vary substantially

Correspondence to: J. B. Gómez (jgomez@ posta.unizar.es) from one fault to another and that, in general, this type of size-frequency relationship is different from the GutenbergRichter law. Many single faults or fault zones display powerlaw distributions only for small events (small compared with the maximum earthquake size a fault can support, given its area), which occur in the intervals between roughly quasiperiodic earthquakes of much larger size which rupture the entire fault. These large and quasi-periodic earthquakes are termed "characteristic" (Schwartz and Coppersmith, 1984), and the resulting size-frequency relationship, Characteristic Earthquake distribution.

There is much debate about the origin of the characteristic earthquake distribution (Dahmen et al., 1998). Because of the short period of instrumental earthquake records and the scarcity of paleoseismic studies (Wesnousky, 1994; Sieh, 1996; Petersen et al., 1996), the statistics of naturally occurring earthquakes in single faults are poor. This fact justifies the development of "synthetic seismicity" models, in which long catalogues of events are generated by computer models of seismogenesis. Such models can be tuned by requiring that they reproduce what is known of the statistics of past seismicity to a reasonable degree, and then use them to forecast statistical inferences about the behaviour of seismicity using much longer and homogeneous catalogues of synthetic events.

Many different seismicity models have been presented in the past twenty years or so. Robinson and Benites (1995) classify these modelling approaches into five groups: (1) cellular automata models, (2) spring-block models, (3) models of single faults in which slip is discretized into patches and obey simplified friction laws, (4) continuum models that utilize realistic constitutive friction laws, and (5) actual physical models.

Cellular automata models appeared in seismological literature in the late 80 s and early 90 s, hand in hand with the concept of self-organized criticality (Bak and Tang, 1989; Ito and Matsuzaki, 1990; Chen et al., 1991; Matsuzaki and Takayasu, 1991; Nakanishi, 1990; Olami et al., 1992). A cellular automaton is a mathematical model of a complex nat- 
ural system which contains a large number of simple identical components with local interactions among themselves (Wolfram, 1994). All cellular automata consist of three basic parts: (i) the underlying lattice, which defines the topology of the spatial discretization, (ii) the neighbourhood, which defines the interaction range between elements; and (iii) the transition rule, which tells us how the state of the system at discrete time $t$ will change at time $t+1$. The link between cellular automata models and seismicity works by discretizing a fault as a one- or two-dimensional plane made up of a large number of patches. These models are usually nondeterministic and neglect the details of both elasticity and fault friction, substituting them by simple cellular automata rules. Despite their simplicity, they are able to reproduce various types of size-frequency statistics, including Gutenberg-Richter and Characteristic Earthquake distributions (Lomnitz-Adler et al., 1992; Barriere and Turcotte, 1994; Main, 1996).

Self-organized criticality (SOC) (Bak et al., 1987, 1988) is a difficult concept to define. In the broadest sense (Sornette, 2000), SOC refers to the spontaneous organization of a large system driven from the outside into a globally stationary state, which is characterized by self-similarity (i.e., power-law) distributions of event sizes and fractal geometrical properties. This stationary state is dynamical in nature and is characterized by statistical fluctuations, which are referred to generically as avalanches. From the seismology point of view, the avalanches are the earthquakes themselves, the external drive is the accumulation of tectonic stress by plate motion, and the self-invariant distribution of event sizes is the Gutenberg-Richter law. Three recent books dealing with SOC in seismology are Turcotte (1999); Sornette (2000); Hergarten (2002), and the reader is referred to these sources for an updated bibliography on the topic.

The key ingredients of any of the seismicity models mentioned above are: (1) the dimensionality of the fault (1D or 2D), (2) the number of faults included in the model (one, a few, or many faults), (3) the employed stress transfer mechanism (nearest-neighbours, long-range elasticity, mean-field), (4) the degree of incorporation of inertial effects (quasistatic, quasi-dynamic, or fully dynamic), (5) the assumed constitutive stress-slip law (experimental, static-dynamic, velocity-weakening, etc.), and (6) the degree of stress conservation (conservative versus dissipative models).

Our purpose here is to build a simple cellular automaton model of seismicity capable of displaying a size-frequency relationship of the Characteristic Earthquake type. That is, a model which exhibits a power-law relationship for small events and an excess of big events (of the order of the system size), together with a very low probability of events of intermediate size. With respect to the six basic ingredients of discrete models of seismicity introduced above, the model presented here is (1) one-dimensional, (2) for a single fault, (3) with a percolation-like stress-transfer mechanism, (4) quasistatic, (5) static/dynamic with total stress drop, and (6) dissipative. To this list we would add that our model is inspired by the concept of asperity (Das and Aki, 1977), i.e. in the presence of a particularly strong element in the system which actually controls its relaxation.

In comparison, the one-dimensional cellular automaton version of the classical Burridge-Knopoff slider-block model has a nearest-neighbour stress-transfer mechanism (ingredient number 3 in the list above) instead of a percolationlike one as our model has. Besides, the cellular automaton Burridge-Knopoff model has one free parameter to be adjusted, which is a ratio of elastic constants and which controls the degree of dissipation of the model (from non conservative to conservative). These two differences clearly separate the behaviour of both models: Gutenberg-Richter for the one-dimensional cellular automaton version of the BurridgeKnopoff model and the Characteristic Earthquake for our model (see Pelletier (2000) for a recent and comprehensive review of the use of spring-block models in seismology).

Also, because of the inherent simplicity of the model, we want to be able to derive analytically some of the statistical properties of the resulting synthetic seismicity using Markov chains.

\section{The model and its simulations}

Consider a one dimensional vertical array of length $N$. The ordered positions, or levels, in the array will be labelled by an integer index $i$ varying from 1 to $N$. This system performs two functions: it is loaded by receiving individual stress particles in the various positions of the array, and unloaded by emitting groups of particles through the first level, $i=1$, which are called relaxations or earthquakes (Fig. 1).

These two functions proceed using the following four rules:

(i) The incoming particles arrive at the system at a constant time rate. Thus, the time interval between each two successive particles will be considered the basic time unit in the evolution of the system.

(ii) All the positions in the array, from $i=1$ to $i=N$, have the same probability of receiving a new particle. When a position receives a particle we say that it is occupied.

(iii) If a new particle comes to a level which is already occupied, its stress is simply dissipated. Thus, a given position $i$ can only be either non-occupied when no particle has come to it, or occupied when one or more particles have come to it.

(iv) The level $i=1$ is special. When a particle goes to this first position a relaxation event occurs. Then, if all the successive levels from $i=1$ up to $i=k$ are occupied, and the position $k+1$ is empty, the effect of the relaxation -or earthquake- is to unload all the levels from $i=1$ up to $i=k$. Hence, the size of this relaxation is $k$, and the remaining levels $i>k$ remain unaltered in their occupancy. 


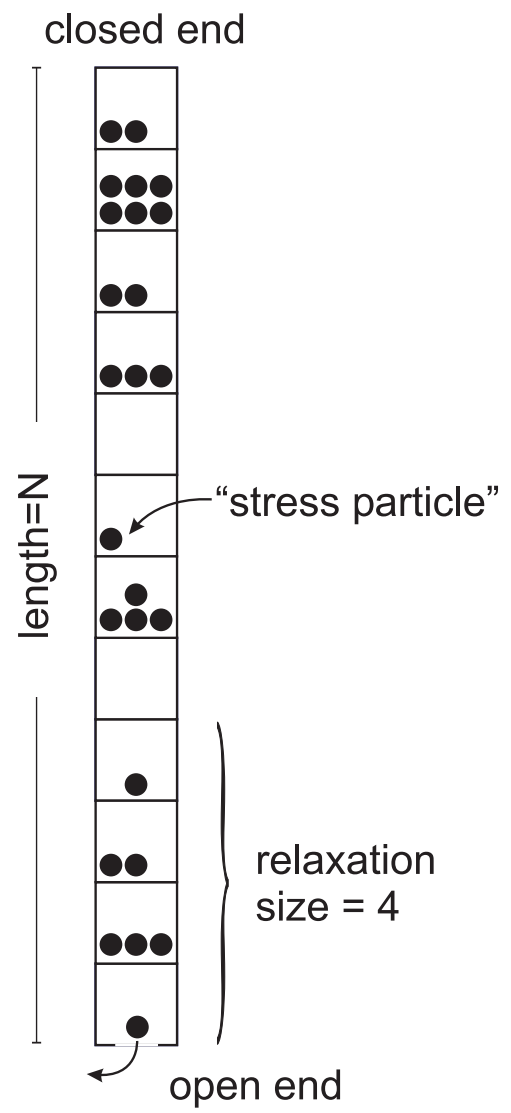

Fig. 1. Layout of the minimalist model. The vertical array of $N$ cells is closed at the top and open at the bottom. Each time step a stress particle is added randomly to the array. A relaxation of size $k$ occurs when the added stress particle hits the lowermost cell. $k$ is the number of consecutive cells, starting from the bottom, that contain at least one stress particle.

Thus, from what has been mentioned above, this model has no parameter; the size $N$ is the unique specification to be made, and the spatial correlation is induced by rule (iv). Now, the state of the system is given by stating which of the $(i>1) N-1$ levels are occupied. Each of these states corresponds to a stable configuration, and therefore the total number of possible configurations is $2^{(N-1)}$. We use the term "total occupancy" for the configuration in which all levels but the first are occupied.

The prominent role given to the level $i=1$ is equivalent to considering that the asperity of the fault is located there. After the occurrence of a relaxation the system is left in a stable configuration. The following particle additions progressively load the system, and when a particle is again assigned to the first level a new relaxation is triggered. Each relaxation empties the lower levels of the system as explained in rule (iv), and the system is left in another stable configuration. The size of the earthquakes can thus range from 1 up to $N$ and the earthquake of maximum size, $k=N$, is called the characteristic one.

From these evolution rules we deduce that after a time unit,

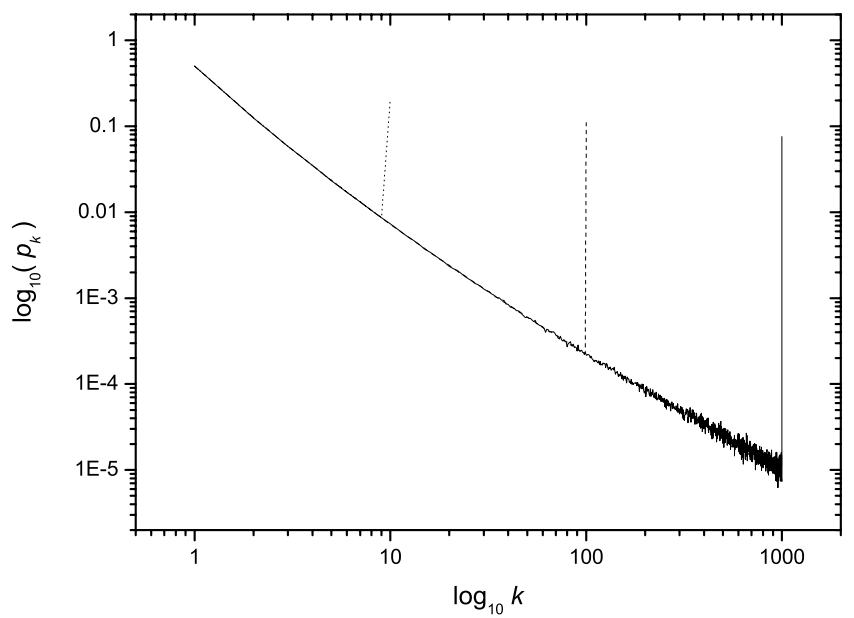

Fig. 2. Probability of occurrence of earthquakes of magnitude $k$. Three simulations are superimposed, corresponding to three different system sizes ( $N=10$ for the dotted line, $N=100$ for the dashed line, and $N=1000$ for the continuous line). Note that the probability of occurrence of earthquakes smaller than the characteristic one is independent of the size of the system, and that the probability of the characteristic earthquake decreases very slowly with $N$.

i.e. after a new incoming particle assignment, we will have an earthquake if the new particle goes to $i=1$, and this occurs with a probability $1 / N$. Conversely, with a probability of $(N-1) / N$ there will be no earthquake. In this case the system will advance one unit in its level of occupation when the new particle is assigned to a non-occupied level, and it will remain at the same configuration if the assigned level was already occupied.

As this model is one-dimensional, extensive Monte Carlo simulations can be performed to accurately explore its properties. In this paper we focus on two important properties: the earthquake size-frequency relation and the statistics of the time of return of the maximum-size earthquake.

The results for the earthquake size-frequency relation, $p_{k}$, are drawn in Fig. 2 and written in Table 1. In Fig. 2 we have superimposed $p_{k}$ for $N=10, N=100$ and $N=1000$. This has been partly included in Table 1 as well. In Fig. 2 there are three notable properties to be commented on. First and most important, we see that the characteristic relaxation, $k=N$, has a much higher probability of occurrence than the big relaxations but with $k<N$. In fact, for $N=10,100$ and 1000, the probability of these three characteristic relaxations does not differ much, and is about $10 \%$. We can express this fact by saying that in this model, grosso modo, one would likely observe only very small earthquakes and the characteristic one. Secondly, forgetting for a moment the case $k=N$, we observe a power law behaviour of the Gutenberg-Richter type for the other earthquakes. The exponent $b$ of this differential distribution is roughly 1.6 (i.e. the corresponding cumulative exponent is compatible with the phenomenological range $0.5 \leq b \leq 1.5$ ). And thirdly, we observe the perfect coincidence of these curves of probability for systems 
Table 1. Probability of occurrence of the earthquake of magnitude $k$ for a different system size, $N$

\begin{tabular}{rccccc}
\hline$k$ & $N=2$ & $N=3$ & $N=4$ & $N=10$ & $N=100$ \\
\hline 1 & 0.500000 & 0.500000 & 0.500000 & 0.500022 & 0.499920 \\
2 & 0.500000 & 0.125000 & 0.125000 & 0.124974 & 0.125130 \\
3 & & 0.375000 & 0.058594 & 0.058599 & 0.058600 \\
4 & & & 0.316406 & 0.034855 & 0.034860 \\
5 & & & & 0.023499 & 0.023460 \\
6 & & & & 0.017126 & 0.017140 \\
7 & & & & 0.013151 & 0.013130 \\
8 & & & & 0.010506 & 0.010510 \\
9 & & & & 0.008627 & 0.008640 \\
10 & & & & 0.208636 & 0.007210 \\
99 & & & & 0.000228 \\
100 & & & & & 0.111320 \\
\hline
\end{tabular}

of different size $N$. This is also appreciated in the numbers collected in Table 1. This, in a sense, unexpected behaviour will be discussed in detail in the next Section.

In Fig. 3 we represent the probability curve for the time of recurrence of the characteristic earthquake. This curve, obtained by Monte Carlo simulations, corresponds to a system of $N=10$. In the abscissas axis, time (denoted by $n$ ) starts at 0 just after the occurrence of a $k=N$ relaxation. It is clear in this model that only after a minimum time lapse of size $N$ the probability of occurrence of a new $k=N$ characteristic earthquake can be non null. We observe in Fig. 3 that after this minimum time lapse, $P(n)$ grows to a maximum and then declines. For the size $N=10$ analyzed in this figure, the maximum of probability corresponds to a time interval $n=34$. This figure agrees with the value obtained later solving the model as a Markov chain process.

\section{The model as a Markov chain}

It is easy to become convinced that, for a given $N$, the $2^{(N-1)}$ stable configurations of our model can be considered as the states of a finite, irreducible and aperiodic Markov chain with a unique stationary distribution (see, for example, Durret, 1999). These configurations are classified into groups according to their occupation number (number of occupied levels); the number of configurations with $j$ occupied levels is $C\left(\begin{array}{c}N-1 \\ j\end{array}\right)$. One step in the chain corresponds to the result of adding a new particle to the system. Up to approximately $N=10$, the transition matrix, $\mathbf{M}$, can be easily obtained using Mathematica as well as the corresponding stationary probabilities for each configuration, which correspond to the components of the eigenvector of $\mathbf{M}$ with eigenvalue, $\lambda$, equal to unity.

For small $N, \mathbf{M}$ and its eigenvectors are obtained by inspection. Let us then start reproducing the first numbers quoted in Table 1 for the probabilities of occurrence of relaxations in small systems. With this aim, let us consider

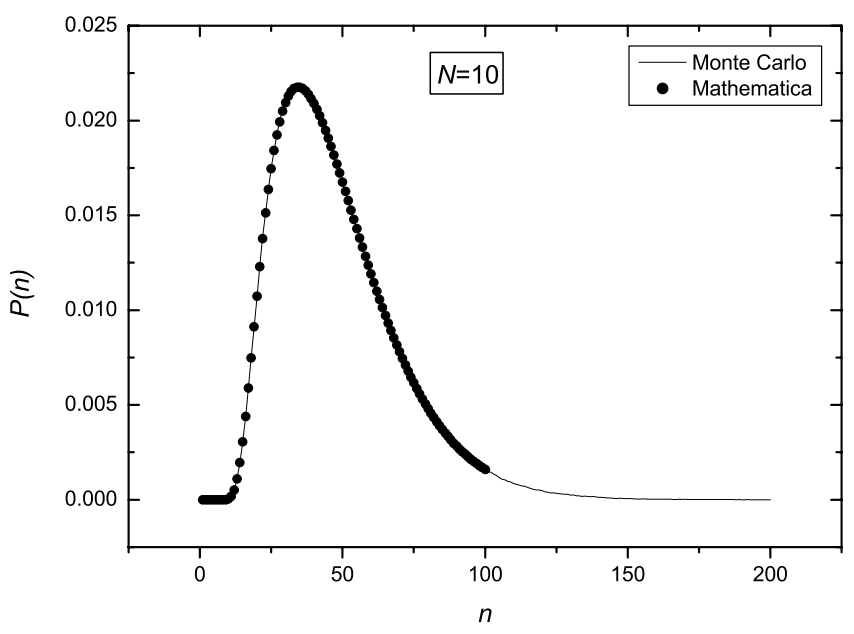

Fig. 3. Probability of return of the characteristic earthquake as a function of time. Time $n$ is the number of stress particles added since the latest relaxation. Note the "stress shadow" for short times, which indicates the existence of a minimum return time between characteristic earthquakes. It is also worth noting the perfect agreement between the Markov chain (dots) and Monte Carlo (line) results.

Fig. 4. In Fig. 4A, B and C there appear all the stable configurations, ordered in an increasing state of occupation, for $N=2, N=3$, and $N=4$, respectively. For the moment, the black level in the top position of the configurations has no meaning.

For $N=2$, using the same order and notation for the configurations as in Fig. 4A, the transition probabilities are $M_{a, a}=1 / 2, M_{a, b}=1 / 2, M_{b, a}=1 / 2$, and $M_{b, b}=1 / 2$. Thus

$\mathbf{M}=\left(\begin{array}{ll}1 / 2 & 1 / 2 \\ 1 / 2 & 1 / 2\end{array}\right), \quad(N=2)$.

This $\mathbf{M}$ is a doubly stochastic matrix and hence the two stationary probabilities are equal.

$p_{a}=1 / 2, \quad p_{b}=1 / 2, \quad(N=2)$.

For $N=3$, the non-null transition probabilities are: $M_{a, a}=$ $1 / 3, M_{a, b}=1 / 3, M_{a, c}=1 / 3 ; M_{b, b}=2 / 3, M_{b, d}=1 / 3$; $M_{c, a}=1 / 3, M_{c, c}=1 / 3, M_{c, d}=1 / 3 ; M_{d, a}=1 / 3$, and $M_{d, d}=2 / 3$. Thus

$\mathbf{M}=\left(\begin{array}{cccc}1 / 3 & 1 / 3 & 1 / 3 & 0 \\ 0 & 2 / 3 & 0 & 1 / 3 \\ 1 / 3 & 0 & 1 / 3 & 1 / 3 \\ 1 / 3 & 0 & 0 & 2 / 3\end{array}\right), \quad(N=3)$.

And the components of the eigenvector corresponding to $\lambda=$ 1 are:

$p_{a}=1 / 4, \quad p_{b}=1 / 4, \quad p_{c}=1 / 8, \quad p_{d}=3 / 8, \quad(N=3)$.

Finally, for $N=4$ the non-null transition probabilities are $M_{a, a}=1 / 4, M_{a, b}=1 / 4, M_{a, c}=1 / 4, M_{a, d}=$ $1 / 4 ; M_{b, b}=2 / 4, M_{b, e}=1 / 4, M_{b, f}=1 / 4 ; M_{c, c}=$ 
A

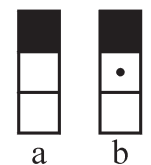

B

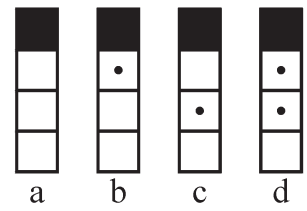

C

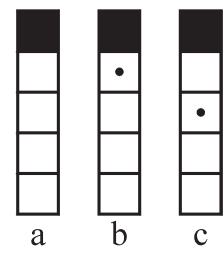

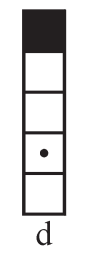
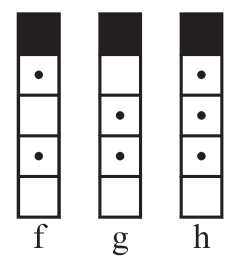

Fig. 4. Set of configurations in systems of size $N=2$ (A), $N=3$ (B), and $N=4(\mathbf{C})$. A dot stands for an occupied cell (i.e., with at least one stress particle). The black box on top of each array is used later in the text to generalize the results in systems of any size. See the text for details.

$2 / 4, M_{c, e}=1 / 4, M_{c, g}=1 / 4, M_{d, a}=1 / 4, M_{d, d}=$ $1 / 4, M_{d, f}=1 / 4, M_{d, g}=1 / 4 ; M_{e, e}=3 / 4, M_{e, h}=$ $1 / 4 ; M_{f, b}=1 / 4, M_{f, f}=2 / 4, M_{f, h}=1 / 4 ; M_{g, a}=$ $1 / 4, M_{g, g}=2 / 4, M_{g, h}=1 / 4 ; M_{h, a}=1 / 4, M_{h, h}=3 / 4$. Thus

$\mathbf{M}=\left(\begin{array}{cccccccc}1 / 4 & 1 / 4 & 1 / 4 & 1 / 4 & 0 & 0 & 0 & 0 \\ 0 & 2 / 4 & 0 & 0 & 1 / 4 & 1 / 4 & 0 & 0 \\ 0 & 0 & 2 / 4 & 0 & 1 / 4 & 0 & 1 / 4 & 0 \\ 1 / 4 & 0 & 0 & 1 / 4 & 0 & 1 / 4 & 1 / 4 & 0 \\ 0 & 0 & 0 & 0 & 3 / 4 & 0 & 0 & 1 / 4 \\ 0 & 1 / 4 & 0 & 0 & 0 & 2 / 4 & 0 & 1 / 4 \\ 1 / 4 & 0 & 0 & 0 & 0 & 0 & 2 / 4 & 1 / 4 \\ 1 / 4 & 0 & 0 & 0 & 0 & 0 & 0 & 3 / 4\end{array}\right), \quad(N=4)$

And, after its diagonalization, one finds

$p_{a}=9 / 64, \quad p_{b}=7 / 64, \quad p_{c}=9 / 128$,

$p_{d}=3 / 64, \quad p_{e}=23 / 128, \quad p_{f}=5 / 64$,

$p_{g}=15 / 256, \quad p_{h}=81 / 256$,

$(N=4)$.

From these numbers, one deduces that in a system with $N=$ 2 levels one should expect relaxations of size $k=1$ with a probability $p_{1}=p_{a}=1 / 2$, and of size $k=2$ with $p_{2}=$ $p_{b}=1 / 2$. In $N=3, p_{1}=p_{a}+p_{b}=1 / 2, p_{2}=p_{c}=1 / 8$, and $p_{3}=p_{d}=3 / 8$. And in $N=4, p_{1}=p_{a}+p_{b}+p_{c}+$ $p_{e}=1 / 2, p_{2}=p_{d}+p_{f}=1 / 8, p_{3}=p_{g}=15 / 256=$ $0.05859 \ldots$, and $p_{4}=p_{h}=81 / 256=0.316406 \ldots$.

Thus, we have observed that in systems with $N=2, N=$ 3 and $N=4$ levels, the value of $p_{1}$ is a constant equal to $1 / 2$ and this result coincides with Table 1 . We have also observed that in $N=3$ and $N=4$ the value of $p_{2}$ is a constant equal

to $1 / 8$, and in $N=4$ we have deduced that $p_{3}=0.05859$. All this agrees with Table 1 .

A conclusive argument proving that in this model the value of $p_{k}$ is a constant, independent of the size $N$, is obtained by re-analyzing Fig. 4 from a wider perspective. Now we consider that in Fig. 4A the configuration labelled by $a$ represents, in a system of size $N$, all the configurations in which the level $i=2$ is not occupied; the rest of the levels $i>2$ are in any possible state of occupation (this is represented by the black level at the top of the configuration). And in Fig. 4A, configuration $b$, we consider a system of size $N$ which has its second level occupied. The probabilities of these two cases must add to unity. Then, defining a Markov chain for these two excluding states, and using the same notation as before, we find $M_{a, a}=(N-1) / N, M_{a, b}=1 / N, M_{b, a}=1 / N$, and $M_{b, b}=(N-1) / N$. The diagonalization of this matrix leads for $p_{a}$ and $p_{b}$ to the same results written in Eq. (3), $p_{a}=p_{b}=1 / 2$. However, its interpretation now is different. Here, $p_{a}=1 / 2$ implies that for any value of $N$, the probability of having a relaxation $k=1$ is $1 / 2$; and $p_{b}=1 / 2$ simply means that the probability of having relaxations $k>1$ is $1 / 2$. Using the same line of reasoning, and referring to Fig. 4B, configuration $a$ represents all the configurations, in a system of size $N$, where levels $i=2$ and $i=3$ are not occupied. And configuration $b$ represents all the configurations where the level $i=2$ is vacant and the level $i=3$ is occupied, etc. Then, the non null transition probabilities are $M_{a, a}=(N-2) / N, M_{a, b}=1 / N, M_{a, c}=1 / N ; M_{b, b}=$ $(N-1) / N, M_{b, d}=1 / N ; M_{c, a}=1 / N, M_{c, c}=(N-$ 2) $/ N, M_{c, d}=1 / N ; M_{d, a}=1 / N, M_{d, d}=(N-1) / N$. The diagonalization of this matrix provides the same stationary probabilities quoted in Eq. (5). Here $p_{c}=1 / 8$ means that for an arbitrary $N$, the probability of occurrence of relaxations of size $2, p_{2}$, is $1 / 8$. And the fact that $p_{a}+p_{b}=1 / 2$ confirms that for any $N, p_{1}=1 / 2$.

Extending this line of reasoning to the 8 configurations drawn in Fig. $4 \mathrm{C}$, one concludes that, for any $N, p_{3}=p g=$ $15 / 256=0.058593$. and one verifies the previous conclusions $p_{1}=1 / 2$ and $p_{2}=1 / 8$.

Therefore, in this model, if for a system of size $N$ one knows all the $p_{k}$ from $k=1$ to $k=N$, then for a system of size $N+1$ the $p_{k}$ are identical, with the exception of the last two, and these fulfill

$p_{N}(N)=p_{N+1}(N+1)+p_{N}(N+1)$.

The recursive way in which $p_{N}(N)$ divides into $p_{N+1}(N+1)$ and $p_{N}(N+1)$ is, however, non trivial.

Let us analyze now, from the Markov-chains point of view, the results for the time of return of the characteristic earthquake shown in Fig. 2. After a $k=N$ relaxation, the system is left in the configuration of no occupancy (for the present discussion we will refer to this configuration as $a_{1}$ ). A new characteristic relaxation will occur when, starting from the configuration $a_{1}$, the system reaches the configuration of total occupancy (which will henceforth be denoted by $a_{N}$ ), and then the next particle is assigned to the $i=1$ level. The number of time steps elapsed between $a_{1}$ and $a_{N}$, plus 1 , will be 
denoted by $n$. And our purpose is to compute the probability of occurrence of a $k=N$ relaxation as a function of $n$. It must be understood that between $a_{1}$ and the occurrence of the next $k=N$ relaxation at time $n$, the system may have visited $a_{N}$ an arbitrary number of times but without triggering any $k=N$ earthquakes. In other words, in those visits there has been no transition from $a_{N}$ to $a_{1}$.

In Markov chains, the transition matrix $\mathbf{M}$ gives the probability of going from one configuration to another in one step, and the $m$-step transition probability is the $m$-th power of $\mathbf{M}$. Thus a simple way to compute $P(n)$ is the following:

1 take $\mathbf{M}$, point to the element in the last row and the first column, and substitute it by 0 . We will denote the new matrix $\mathbf{M}^{\prime}$;

2 compute $\mathbf{T}_{n}=\mathbf{M}^{(n-1)}$;

3 take the element of the first row and the last column of $\mathbf{T}_{n}$, and multiply it by $1 / N$. This result is $P(n)$.

This is clear, because $\mathbf{M}^{\prime}$ does not permit transitions from $a_{N}$ to $a_{1}$. Thus, in $\mathbf{T}_{n}$ we have all the probabilities of transitions, in $n-1$ steps, between all the configurations, with the restriction that from $a_{N}$ to $a_{1}$ this passing is forbidden. Hence, in $\mathbf{T}_{n}$ the matrix element of the first row and the last column corresponds to the transition from $a_{1}$ to $a_{N}$ in $n-1$ steps and with the mode $a_{N} \longrightarrow a_{1}$ locked. Finally, as the $1 / N$ factor is the probability of the transition $a_{N} \longrightarrow a_{1}$, we actually have built $P(n)$. In Fig. 3, for $N=10$, we see the perfect match between the Monte Carlo simulations and the results coming from the theory of Markov, calculated using Mathematica.

In order to get a quantitative insight on $P(n)$, let us apply this method to $N=2$. In this case, $\mathbf{M}$ is given by Eq. (2). Hence,

$\mathbf{M}^{\prime}=\left(\begin{array}{cc}1 / 2 & 1 / 2 \\ 0 & 1 / 2\end{array}\right)$,

$\mathbf{M}^{\prime(n-1)}=\left(\frac{1}{2}\right)^{(n-1)}\left(\begin{array}{cc}1 & n-1 \\ 0 & 1\end{array}\right)$,

and, therefore,

$P(n)=(n-1)(1 / 2)^{(n-1)}(1 / 2)=\frac{n-1}{2^{n}}, \quad(N=2)$.

Thus, we observe that the asymptotic fall-off behaviour of $P(n)$ is of the type

$P(t) \propto t \exp (-t), \quad(N=2)$.

It is important to recall that in aperiodic, irreducible and finite Markov Chains such as that of our model, the mean waiting time for a configuration is the inverse of the stationary probability of that configuration. Then, the mean time between characteristic relaxations in this model is

$<n>=\frac{1}{\frac{a_{N}}{N}}=\frac{N}{a_{N}}$.

As an example, for $N=4,<n>=4 \cdot(256 / 81)=12.4$ time units.

\section{Discussion and conclusions}

We have presented a one-dimensional discrete model of seismicity that, inspired by the role of an asperity in a fault, displays a size-frequency spectrum similar to that expected from Characteristic Earthquake behaviour. Although this stochastic model obviously lacks the explicit physics of other detailed -and complex-dynamical models of earthquakes, its basic hypotheses and implications are clear, phenomenologically reasonable and coherent. The size invariance of this model, also surprisingly manifests itself in predicting an identical probability of occurrence for earthquakes of the same magnitude independent of the size $N$ of the system. In this universal rule the characteristic earthquake is excluded. This model has the additional bonus that several important predictions can be algebraically derived by using the theoretical framework of the Markov chains. Specifically, the statistics of the time of return of the characteristic earthquake are neatly predicted by this formalism. We are currently working on the comparison of the predicted return time of the characteristic earthquake in this model with actual return times for earthquakes in active faults. A preliminary analysis shows that real characteristic earthquakes on faults have a behaviour consistent with the prediction of our model (Gonzáles et al., 2002). These results will be published elsewhere.

Dahmen et al. (Dahmen et al., 1998) report a model able to pass from the Gutenberg-Richter to the Characteristic Earthquake behaviour and, what is more interesting, these authors define a configurational entropy as an appropriate concept that reflects which of these two extreme behaviours the system is actually operating in. In qualitative terms, the Gutenberg-Richter behaviour corresponds to a high entropy mode of operation while the Characteristic Earthquake behaviour corresponds to a low entropy mode. Therefore, we would like to make an assesment of our model from this configurational entropy point of view and check the concordance, or not, with the conclusions of Ref. Dahmen et al. (1998).

In our model, the configurations are classified into groups according to the number of levels, $j$, that are occupied. The statistical weight of each $j$ is $C\left(\begin{array}{c}N-1 \\ j\end{array}\right)$, which has its maximum values for $j$ about $N / 2$. Conversely, the statistical weight is minimum on the extrema: for $j$ near 1 and for $j$ near $N-1$. Then, we need to find out the values of the stationary probabilities of each occupation number $j$. This is shown in Fig. 5 for a system of size $N=100$. There we observe how in our model the system resides most of the time in the configurations of maximum occupancy, that is, where the configurational entropy is a minimum, agreeing with the interpretation of Ref. Dahmen et al. (1998).

As a final minor remark, note that in Fig. $5 p_{(j=N)}$ and $p_{(j=N-1)}$ are identical. This is a property that holds in this model for any value of $N$ and which can be easily proved. For brevity reasons, we omit this proof.

Acknowledgements. Amalio F. Pacheco and Miguel VázquezPrada dedicate this paper to Professor Domingo González on the 


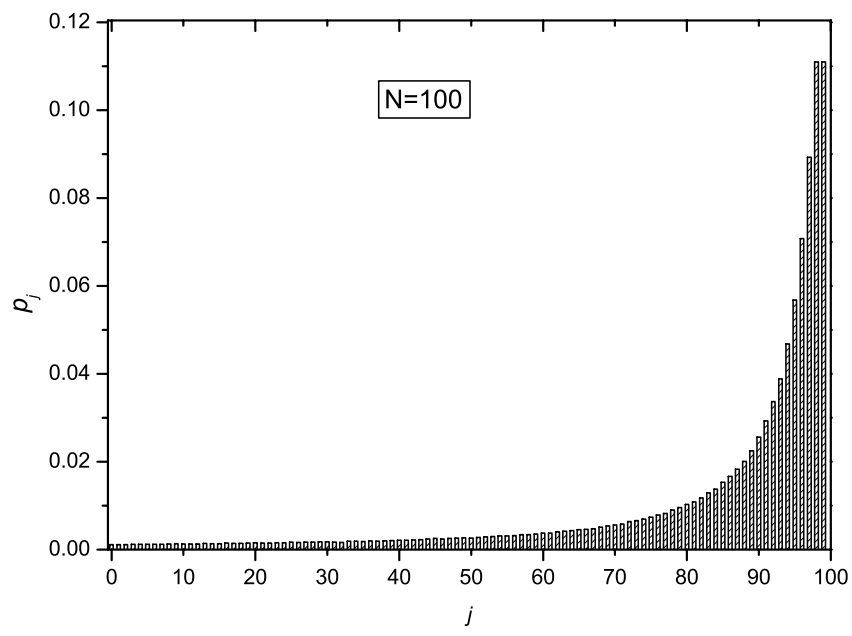

Fig. 5. Stationary probabilities $p_{j}$ as a function of the number of levels occupied, $j$ in a system of size $N=100$. The system spends most of the time in configurations with a large fraction of its cells occupied, conferring to the system a very low configurational entropy.

occasion of his retirement from the Physics Department of the University of Zaragoza.

AFP thanks Jesús Asín, Jorge Ojeda, Carmen Sangüesa and Miguel San Miguel for many fruitful discussions. JBG thanks Ian Main of the University of Edinburgh for enlightening comments and suggestions. This work was supported by the Spanish DGICYT (Project BFM2002-01798). MVP and AG are supported by PhD research grants B037/2001 and B013/2002 funded by the Autonomous Government of Aragón (DGA) and the European Social Fund.

\section{References}

Bak, P. and Tang, C.: Earthquakes as a self-organized critical phenomenon, J. Geophys. Res., 94, 15 635-15 637, 1989.

Bak, P., Tang, C., and Wisenfeld, K.: Self-organized criticality: an explanation of 1/f noise, Phys. Rev. Lett., 59, 381-384, 1987.

Bak, P., Tang, C., and Wisenfeld, K.: Self-organized criticality, Phys. Rev. A, 38, 364-374, 1988.

Barriere, B. and Turcotte, D. L.: Seismicity and self-organized criticality, Phys. Rev. E, 49, 1151-1160, 1994.

Chen, K., Bak, P. and Obukhov, S. P.: Self-organized criticality in a crack propagation model of earthquakes, Phys. Rev. A, 43, 625630, 1991.

Dahmen, K., Ertas, D., and Ben-Zion, Y.: Gutenberg-Richter and characteristic earthquake behavior in simple mean-field models of heterogeneous faults, Phys. Rev. E, 58, 1494-1501, 1998.

Das, S. and Aki, K.: Fault plane with barriers: a versatile earthquake model, J. Geophys. Res., 82, 5658-5670, 1977.

Durrett, R.: Essentials of Stochastic Processes, Springer Verlag, Berlin, Germany, pp. 304, 1999.

González, Á., Gómez, J. B., Vázquez-Prada, M., and Pacheco, A. F.: Scaling of characteristic earthquake return interval with nor- malized fault length: data and a minimalist model, Geophys. Res. Abstr., 4, Abstract number EGS02-A-02967, 2002.

Gutenberg, B. and Richter, C. F.: Magnitude and energy of earthquakes, Ann. Geofis., 9, 1-15, 1956.

Hergarten, S.: Self-Organized Criticality in Earth Systems. 272 pp., Springer Verlag, Berlin, Germany, 2002.

Ito, K. and Matsuzaki, M.: Earthquakes as self-organized critical phenomena, J. Geophys. Res., 95, 6853-6860, 1990.

Kagan, Y. Y.: Universality of the seismic moment-frequency relation, Pure Appl. Geophys., 155, 537-573, 1999.

Kanamori, H. and Anderson, D. L.: Theoretical basis of some empirical relations in seismology, Bull. Seismol. Soc. Am., 65, 1073-1095, 1975.

Knopoff, L.: The magnitude distribution of declustered earthquakes in Southern California, Proc. Natl. Acad. Sci. USA, 97, 11 880$11884,2000$.

Lomnitz-Adler, J., Knopoff, L., and Martínez-Mekler, G.: Avalanches and epidemic models of fracturing in earthquakes, Phys. Rev. A, 45, 2211-2221, 1992.

Main, I.: Statistical physics, seismogenesis, and seismic hazard, Rev. Geophys., 34, 433-462, 1996.

Matsuzaki, M. and Takayasu, H.: Fractal features of the earthquake phenomenon and a simple mechanical model, J. Geophys. Res. 96, 19925-19931, 1991.

Nakanishi, H.: Cellular-automaton model of earthquakes with deterministic chaos, Phys. Rev. A, 41, 7086-7089, 1990.

Olami, Z., Feder, J. S., and Christensen, K.: Self-organized criticality in a continuous, nonconservative cellular automaton modeling earthquakes, Phys. Rev. Lett., 68, 1244-1247, 1992.

Pelletier, J. D.: Spring-block models of seismicity: review and analysis of a structurally heterogeneous model coupled to a viscous asthenosphere, in: Geocomplexity and the Physics of Earthquakes, Geophysical Monographs, 120, American Geophysical Union, Washington, USA, 27-42, 2000.

Petersen, M. D., Bryant, W. A., Cramer, C. H., Cao, T., Reichle, M., Frankel, A. D., Lienkaemper, J. J., McCrory, P. A., and Schwartz, D. P.: Probabilistic Seismic Hazard Assessment for the State of California. California Department of Conservation's Division of Mines and Geology Open-File Report 96-08, and United States Geological Survey Open-File Report 96-706, pp. 66, 1996.

Robinson, R. and Benites, R.: Synthetic seismicity models of multiple interacting faults, J. Geophys. Res., 100, 18 229-18 238, 1995.

Schwartz, D. P. and Coppersmith, K. J.: Fault behavior and characteristic earthquakes: examples from the Wasatch and San Andreas fault zones, J. Geophys. Res., 89, 5681-5698, 1984.

Sieh, K.: The repetition of large earthquake ruptures, Proc. Natl. Acad. Sci. USA, 93, 3764-3771, 1996.

Sornette, D.: Critical Phenomena in Natural Sciences, Springer Verlag, Berlin, Germany, pp. 434, 2000.

Turcotte, D. L.: Fractals and Chaos in Geology and Geophysics, Cambride University Press, Cambridge, UK, pp. 300, 1999.

Wesnousky, S. G.: The Gutenberg-Richter or characteristic earthquake distribution, which is it? Bull. Seismol. Soc. Am., 84, 1940-1959, 1994.

Wolfram, S.: Cellular automata: collected papers, Addison-Wesley, New York, USA, pp. 596, 1994. 